\title{
Acceptance Angle Control for Improved Transmission Imaging in an SEM
}

\section{Jason Holm* and Robert R. Keller}

National Institute of Standards and Technology, Applied Chemicals and Materials Division, 325 Broadway, Boulder, CO

*jason.holm@nist.gov

\begin{abstract}
This contribution presents a simple, cost-effective modular aperture system enabling comprehensive acceptance angle control for STEM-in-SEM imaging. The system is briefly described, and different ways to use it are explained. To demonstrate the utility of the approach, a few samples are examined using the new system with comparisons to images from traditional SEM detectors. We show that the system enables conventional STEM imaging modes ranging from brightfield to high-angle annular darkfield (that is, Z-contrast), thin annular detection schemes, and even some non-conventional imaging modes.
\end{abstract}

\section{Introduction}

Recent years have seen rapid growth of transmitted electron imaging techniques within the scanning electron microscope (SEM), representing the emergence of the field known as "transmission SEM," or t-SEM [1]. While not entirely new, the resurgence is due in part to recent detector technology advances. Combined sample holder/detectors as well as stand-alone segmented detectors, similar to those used in conventional high-energy scanning transmission electron microscopy (STEM) [2-6], lie at the heart of these advances. In fact, all major SEM manufacturers and accessory vendors now offer various STEM detectors as optional add-ons. As a group, these detectors and their associated imaging techniques are often termed "STEM-in-SEM" or "low-voltage STEM."

Despite the demonstrated utility of t-SEM imaging [7], the full promise of the technique has not yet been realized. One restriction is that commercial transmitted electron detectors do not allow users to easily control acceptance angles and select which scattered electrons contribute to images. In conventional SEMs there are no post-specimen lenses to focus electrons, so the desired acceptance angles must be obtained through a combination of detector geometry and camera length (CL). To that end, some commercial STEM detectors consist of arrays that include a central circular element for brightfield (BF) imaging and two or three concentric annular elements to detect electrons forward-scattered through different angular ranges for darkfield (DF) imaging. Although these arrays enable some acceptance angle control, comprehensive control is key to extracting the maximum amount of information from any detector. Area detectors with multiple sensors or direct electron detectors may be promising techniques for selecting electrons forward-scattered through specific angles [8], but they are not yet commercially available for SEMs.

This article demonstrates an economical way to obtain comprehensive acceptance angle control using a commercially available STEM detector with little built-in angular selectivity. The strategy involves a new modular aperture system and a new sample holder, which together can enable most conventional STEM imaging modes in any SEM capable of accepting a transmitted electron detector. The approach is not specifically limited to STEM-in-SEM but could be applied to other microscopes and devices that select electrons or other particles scattered through different angles.

\section{Materials and Methods}

Materials. Several sample types were imaged to demonstrate the aperture system utility: bundled single-wall carbon nanotubes (SWCNT) with catalyst particles, multi-wall carbon nanotubes (MWCNT) without catalyst particles, $\mathrm{Au}$ and $\mathrm{TiO}_{2}$ nanoparticles, and exfoliated two-dimensional (2D) zeolites. Mild sonication was used to disperse the carbon nanotubes and nanoparticles in different solvents (SWCNTs in chloroform, MWCNTs in n-methylpyrrolidone, and $\mathrm{Au}$ and $\mathrm{TiO}_{2}$ particles in ethanol.) A small amount of each dispersion was drop-cast onto lacey carbon support grids and allowed to dry in air. Zeolites were prepared elsewhere [9] and were deposited on an ultrathin carbon/lacey carbon substrate.
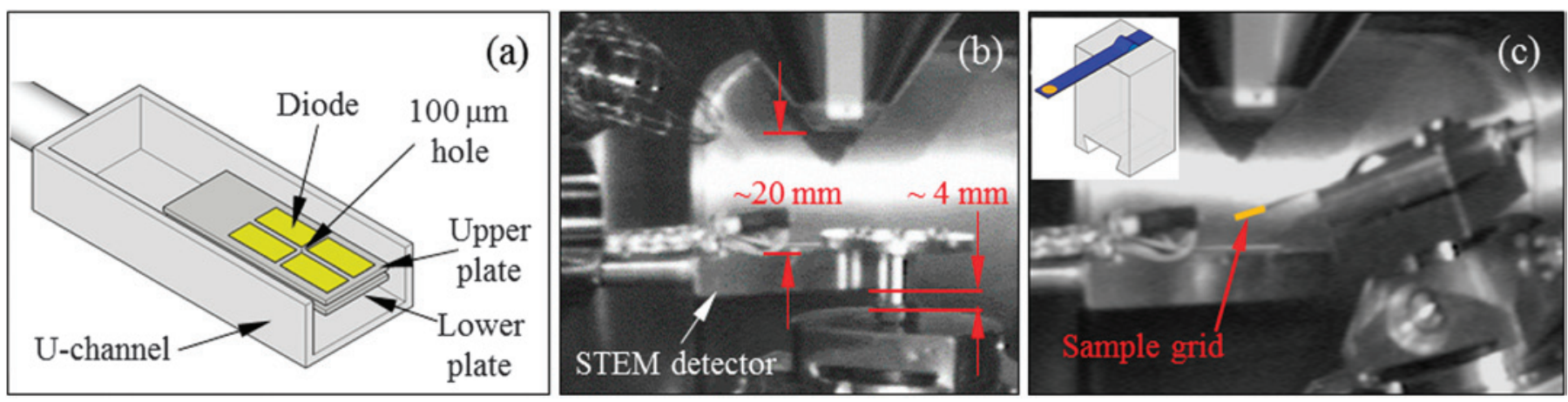

Figure 1: (a) An illustration of the STEM detector showing the diode geometry and layout. (b) An interior view of the SEM chamber showing the detector at its lowest position with the vendor-supplied carousel style holder demonstrating the limited sample maneuverability ( $4 \mathrm{~mm}$ vertical). (c) An interior view of the SEM chamber showing a new cantilever-style holder for positioning a sample at arbitrary orientation. The inset shows an illustration of the holder on a dove-tail standoff block. 


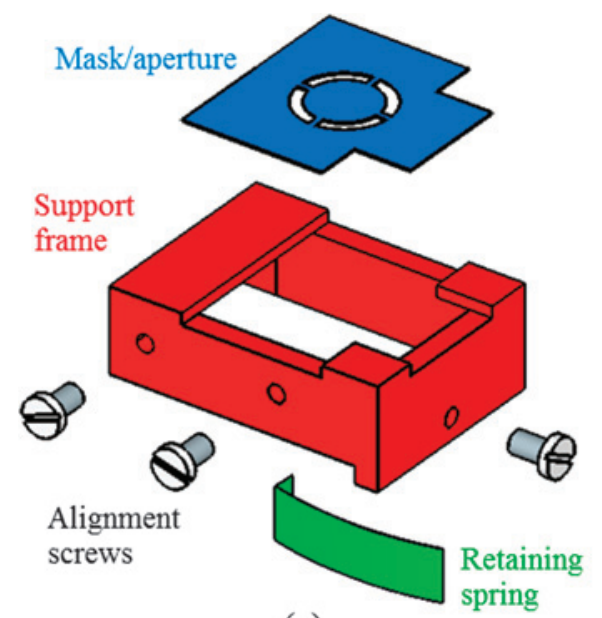

(a)

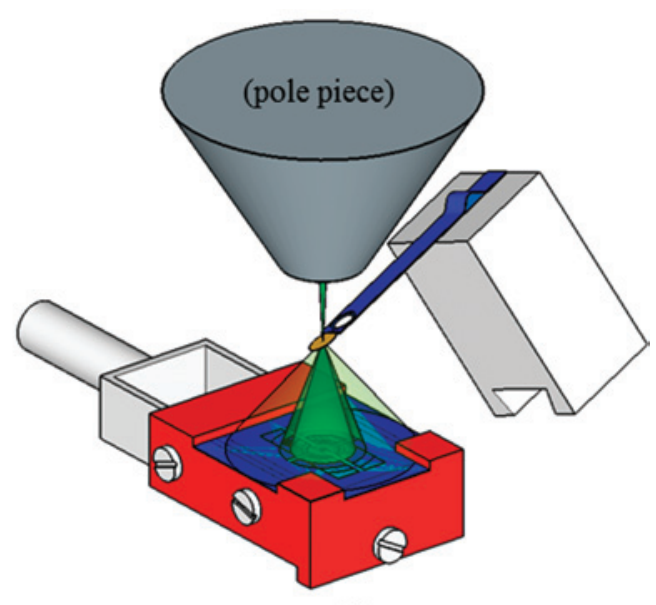

(b)

Figure 2: (a) An illustration of the modular aperture system components, and (b) an assembly view including the STEM detector and the new cantilever-style sample holder with a sample at an arbitrary orientation. Primary electrons are shown emerging from the SEM pole-piece and scattering in a forward direction through the sample and to the detector.

STEM detector design. A Zeiss LEO 1525 SEM equipped with a Schottky field emission electron gun was used to image the samples at $30 \mathrm{kV}$ with a $30 \mu \mathrm{m}$ condenser aperture, resulting in a spot size of $4-5 \mathrm{~nm}$ and a probe current $\sim 165 \mathrm{pA}$. Detectors used here included a KE-Developments STEM detector, an Everhart-Thornley secondary electron (SE) detector, and an ETP Semra Series 8.6 Robinson backscattered electron (BSE) detector.

The new STEM detector comprises two plates (Figure 1a): an upper plate for DF imaging with four rectangular diodes surrounding a $100 \mu \mathrm{m}$ diameter through-hole and a lower plate with a diode for BF imaging positioned under the through-hole. Note that angular selectivity built into this detector is minimal, and acceptance angle adjustments must be obtained through changes in CL, defined here as the distance between the sample and the detector diode. The STEM detector also has an $x y z$-positioning stage to align the diodes with the optic axis. This detector positioning feature can also be used to elicit unconventional and potentially useful image contrast.

One step toward comprehensive acceptance angle control involves moving the detector to an appropriate distance from the pole piece. For example, when the transmitted electron detector is at its lowest position (Figure 1b), the distance between the top of the detector and the bottom of the pole piece is $\sim 20 \mathrm{~mm}$, thereby maximizing the available CL and space for positioning the sample. When the transmitted electron detector is set at its highest position, the distance between the detector and pole piece is $\sim 10 \mathrm{~mm}$.

Cantilever specimen holder. Positioning a sample at any location and/or orientation between the detector and the pole piece is also essential for comprehensive STEM-in-SEM imaging. A new cantilever-clamp-style sample holder [10] can be used for that purpose (Figure 1c). In addition to allowing precise sample positioning, the cantilevers can be made very thin, allowing the sample to be located almost anywhere in the vacant space between the STEM detector and pole piece. existing $100 \mu \mathrm{m}$ through-hole and the carousel-style holder is $\sim 10<\beta<25 \mathrm{mrad}$. Substitution of the cantilever-style holder enables a BF range of $\sim 2.5<\beta<50$ mrad. For DF imaging with the vendor-supplied carousel-style holder, the acceptance half-angle range is $\sim 85<\beta<1270 \mathrm{mrad}$. Substitution of the cantilever-style holder expands that range to $\sim 20<\beta<1420 \mathrm{mrad}$. The minimum inner acceptance half-angle $\left(\beta_{\mathrm{i}} \approx 20 \mathrm{mrad}\right)$ is due to a small gap between the diodes and the $100 \mu \mathrm{m}$ through-hole. However, when a single diode is used, $\beta$ can be much smaller than $20 \mathrm{mrad}$, thereby enabling marginal and annular BF imaging.

Perhaps the key step toward comprehensive acceptance angle control is the mask/aperture system. One embodiment of the system includes two main components: (1) a support frame

Table 1: STEM signal collection modes and their associated acceptance angles. The primary electron beam convergence semi-angle is $\alpha$, and the inner and outer acceptance semi-angles are $\beta_{\mathrm{i}}$ and $\beta_{\circ}$ (see Figure 3a). Note that these definitions are not all-encompassing and that other definitions may exist.

\begin{tabular}{|l|l|}
\hline $\begin{array}{l}\text { STEM-in-SEM } \\
\text { Signal Collection Mode }\end{array}$ & Acceptance Angle Range \\
\hline Brightfield (BF) & $\beta_{\mathrm{i}}=0, \beta_{\mathrm{o}}<\alpha$ \\
\hline Annular Brightfield (ABF) & $0<\beta_{\mathrm{i}}, \beta_{\mathrm{o}}<\alpha$ \\
\hline Marginal Brightfield (MBF) & $\beta_{\mathrm{i}} \approx \alpha$, \\
\hline Thin Annular Detector (TAD) & $\beta_{\mathrm{o}} \approx 1.1 \beta_{\mathrm{i}}$ \\
\hline Low-Angle Annular & $\beta_{\mathrm{i}}>\alpha$ \\
Darkfield (LAADF) & $\beta_{\mathrm{o}} \leq 50 \mathrm{mrad}$ \\
\hline Medium-Angle Annular & $\beta_{\mathrm{i}}>50 \mathrm{mrad}$ \\
Darkfield (MAADF) & $\beta_{\mathrm{o}}<100 \mathrm{mrad}$ \\
\hline High-Angle Annular & \\
Darkfield (HAADF) & $\beta_{\mathrm{i}} \geq 100 \mathrm{mrad}$ \\
\hline
\end{tabular}

*LAADF, MAADF, and HAADF distinctions are somewhat arbitrary, and the ranges provided are typically associated with high-energy STEM. Because lower-energy electrons scatter more strongly, these values will be somewhat higher for STEM-in-SEM. 


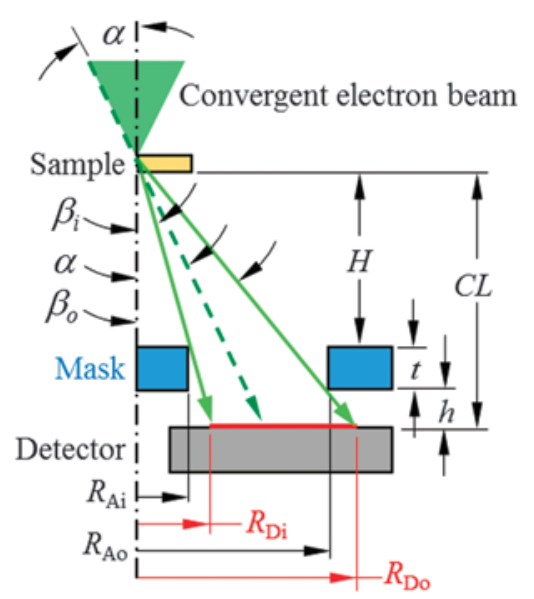

(a)

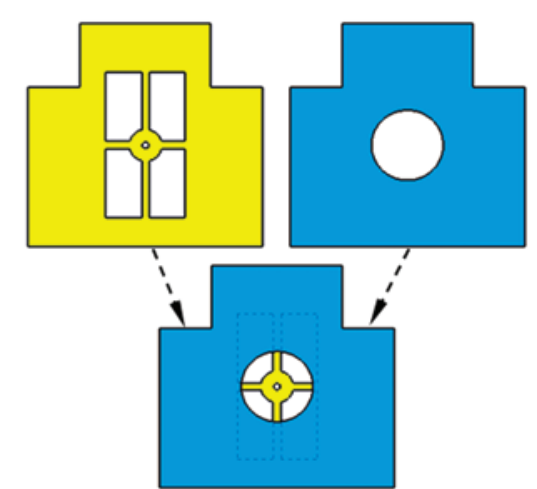

(b)

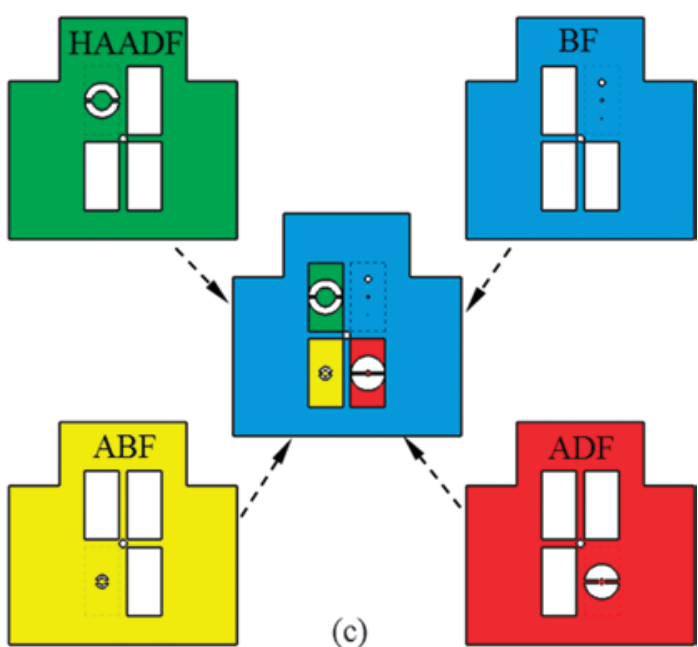

(c)

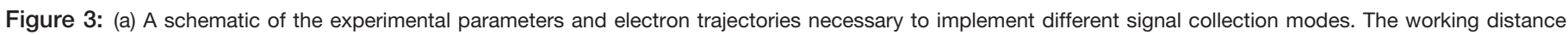

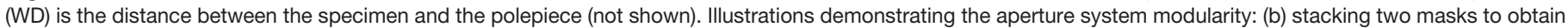

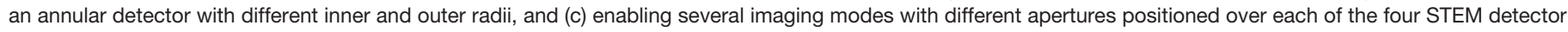
diodes.

and a mask/aperture (Figure 2a) that can be adapted to most commercially available STEM detectors and (2) the cantileverstyle holder shown in Figure $2 \mathrm{~b}$ at an arbitrary orientation with an edge-clamped sample.

Signal collection modes. Signal collection modes, described in Table 1 and shown schematically in Figure 3a with the optic axis and a mask centered over a single diode, can be implemented by using masks in different ways. For example, a variable-annulus aperture scheme can be implemented by stacking masks with different apertures (Figure $3 \mathrm{~b}$ ). The mask on the upper left can be used alone to exclude small-angle scattering from DF images, or it can be stacked with the mask on the upper right to admit electrons scattered through a specific angular range. Note that $\mathrm{BF}$ imaging can still be implemented with the lower detector plate by including a small aperture in the center of the masks.

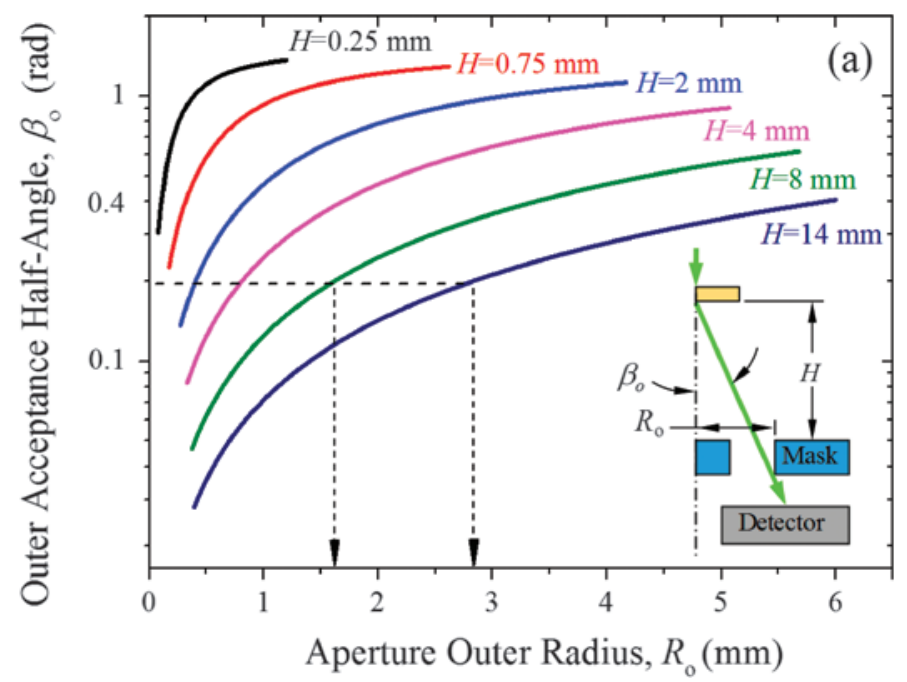

The four rectangular detector diodes provide much utility when combined with the integral $x y z$-positioning stage. For example, if the detector stage is used to align individual diodes with the optic axis, each of those diodes can be used for different imaging modes when appropriate apertures are employed (Figure 3c), provided that off-axis detector elements can be disabled or masked. A significant advantage to locating apertures over individual detector diodes is that very small acceptance angles can be selected. In this way, the aperture system enables signal collection modes beyond basic $\mathrm{BF}$ and DF imaging (Table 1): BF imaging with apertures other than the existing $100 \mu \mathrm{m}$ through-hole, annular brightfield imaging [11], thin annular detection schemes [12], marginal brightfield imaging akin to that described by Cowley [13] in which a thin annular detector collects electrons scattered into acceptance angles straddling the beam convergence angle,

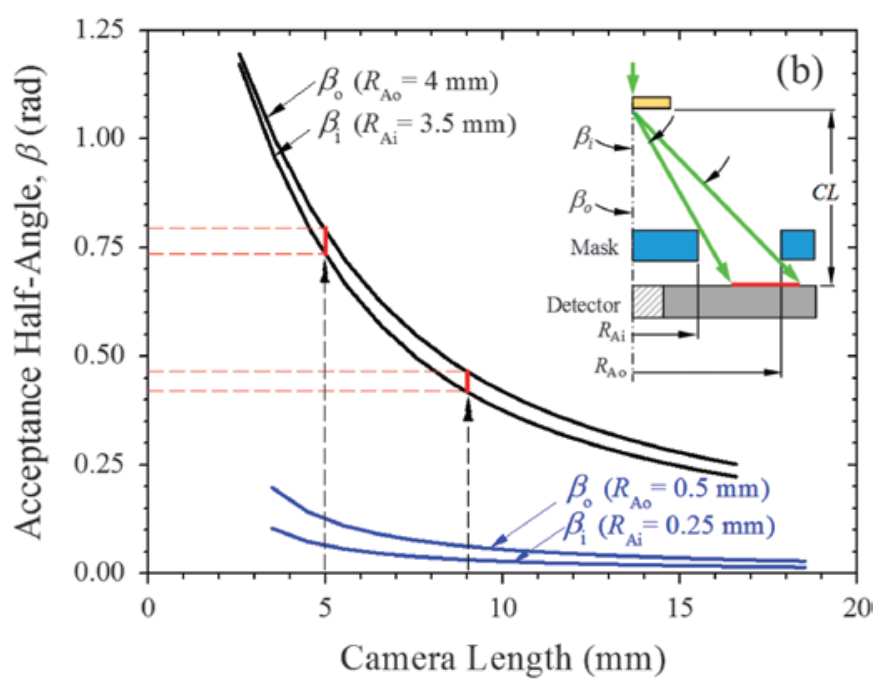

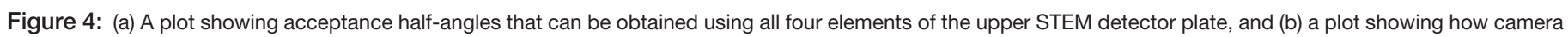

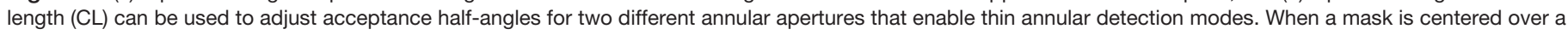
single detector element, the box representing the detector should be extended to the optic axis. 

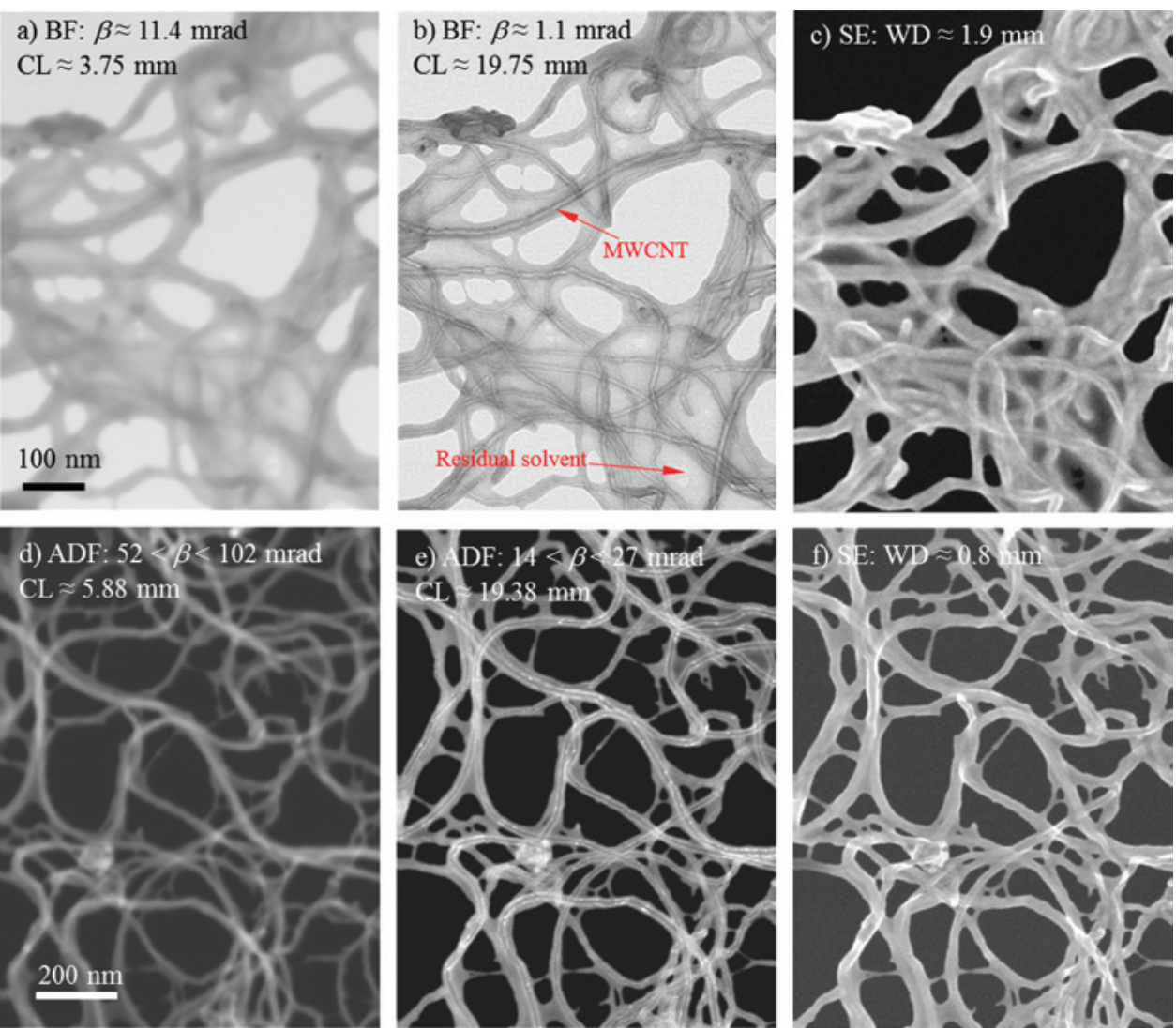

Figure 5: Micrographs of MWCNTs in residual organic solvent imaged with different detectors. BF STEM images were recorded using a $20 \mu \mathrm{m}$ diameter aperture, and DF STEM images were taken with an annular aperture $\left(R_{\mathrm{Ai}}=0.25 \mathrm{~mm}, R_{\mathrm{Ao}}=0.5 \mathrm{~mm}\right)$ centered over a single diode. (a) $\mathrm{BF}$ image $(\mathrm{CL}=3.75 \mathrm{~mm}, \mathrm{WD}=18.2 \mathrm{~mm}),(\mathrm{b}) \mathrm{BF} \mathrm{image}$ $(C L=19.75 \mathrm{~mm}, W D=1.9 \mathrm{~mm}),(\mathrm{c}) \mathrm{SE}$ image $(\mathrm{WD}=1.9 \mathrm{~mm}),(\mathrm{d}) \mathrm{ADF}$ image $(C L=5.88 \mathrm{~mm}, \mathrm{WD}=14.2 \mathrm{~mm}),(\mathrm{e}) \mathrm{ADF}$ image $(C L=19.38 \mathrm{~mm}, W D=0.8 \mathrm{~mm})$, and $(f) S E$ image $(W D=0.8 \mathrm{~mm})$.

as well as annular darkfield (ADF) imaging at low, medium [14], and high angles [15].

Example setups. Selecting and implementing specific signal collection modes is straightforward with the aperture system. The basic procedure is to choose a mask with specific aperture dimensions and then use the SEM sample positioning stage to adjust the $\mathrm{CL}$ and admit electrons scattered into desired acceptance angle ranges. For example, Figure 4 a shows how $\beta_{\mathrm{o}}$ varies with aperture radius $R_{\mathrm{o}}$ and $H$, the sample-to-mask distance. Note that $\mathrm{CL}=H+t+h$, where $t$ is the mask thickness, and $h$ is the mask-to-detector distance. As the figure indicates, $\beta_{\mathrm{o}}=200 \mathrm{mrad}$ can be obtained using all four diodes with different apertures (that is, with $R_{\mathrm{o}} \approx 1.6 \mathrm{~mm}$ and $H=8 \mathrm{~mm}$, or with $R_{\mathrm{o}} \approx 2.8 \mathrm{~mm}$ and $H=14 \mathrm{~mm}$ ). Acceptance angles can also be shifted around the desired values by using the sample positioning stage to change the CL. Figure $4 \mathrm{~b}$ shows how two different annular apertures can enable thin annular detector configurations to select electrons scattered through different angles. The black lines encompass acceptance angles accessible with a large aperture (inner radius $R_{\mathrm{Ai}}=3.5 \mathrm{~mm}$, outer radius $R_{\mathrm{Ao}}=4 \mathrm{~mm}$ ) centered over all four diodes; the blue lines encompassacceptance angles accessiblewith a smaller aperture $\left(R_{\mathrm{Ai}}=0.25 \mathrm{~mm}, R_{\mathrm{Ao}}=0.5 \mathrm{~mm}\right)$ located over a single diode. As the figure indicates, a large acceptance angle range can $5 \mathrm{~b}$ show $\mathrm{BF}$ images recorded using a mask with a $20 \mu \mathrm{m}$ diameter aperture, and Figure $5 \mathrm{c}$ shows a conventional SE image. Figures $5 \mathrm{~d}$ and $5 \mathrm{e}$ show ADF images recorded using a mask with an annular aperture $\left(R_{\mathrm{Ai}}=0.25 \mathrm{~mm}, R_{\mathrm{Ao}}=0.5 \mathrm{~mm}\right)$ centered over one of the STEM detector diodes, and Figure $5 f$ shows a conventional SE image recorded simultaneously with $5 \mathrm{e}$. Individual nanotubes are discernable in $5 \mathrm{a}$ and $5 \mathrm{~b}$. In Figure 5b, however, bends and other deformations along many tubes can be observed, and inner and outer tube diameter measurements are feasible. Tubes are discernable in $5 \mathrm{c}$, but diameter measurements are not feasible because of the residual solvent. In Figures 5d and 5f, many MWCNTs are visible, but they are difficult to differentiate from the residual solvent. Nanotubes in Figure 5e, however, can be differentiated from solvent because bright lines delineate the MWCNTs. Note that $\beta_{i}=14 \mathrm{mrad}$ is sufficiently small to capture Bragg-scattered electrons, and therefore it is feasible that the bright lines are partially due to diffraction. It is unclear why Figures $5 \mathrm{a}$ and $5 \mathrm{~d}$ exhibit poor resolution compared to the other images, but the reduced resolution is consistently observed at shorter camera lengths with or without the aperture system in place.

$\mathrm{Au}$ and $\mathrm{TiO}_{2}$ particles on lacey carbon. BSE detectors are indispensable for numerous applications, but for sufficiently thin samples where atomic number contrast is desired, 

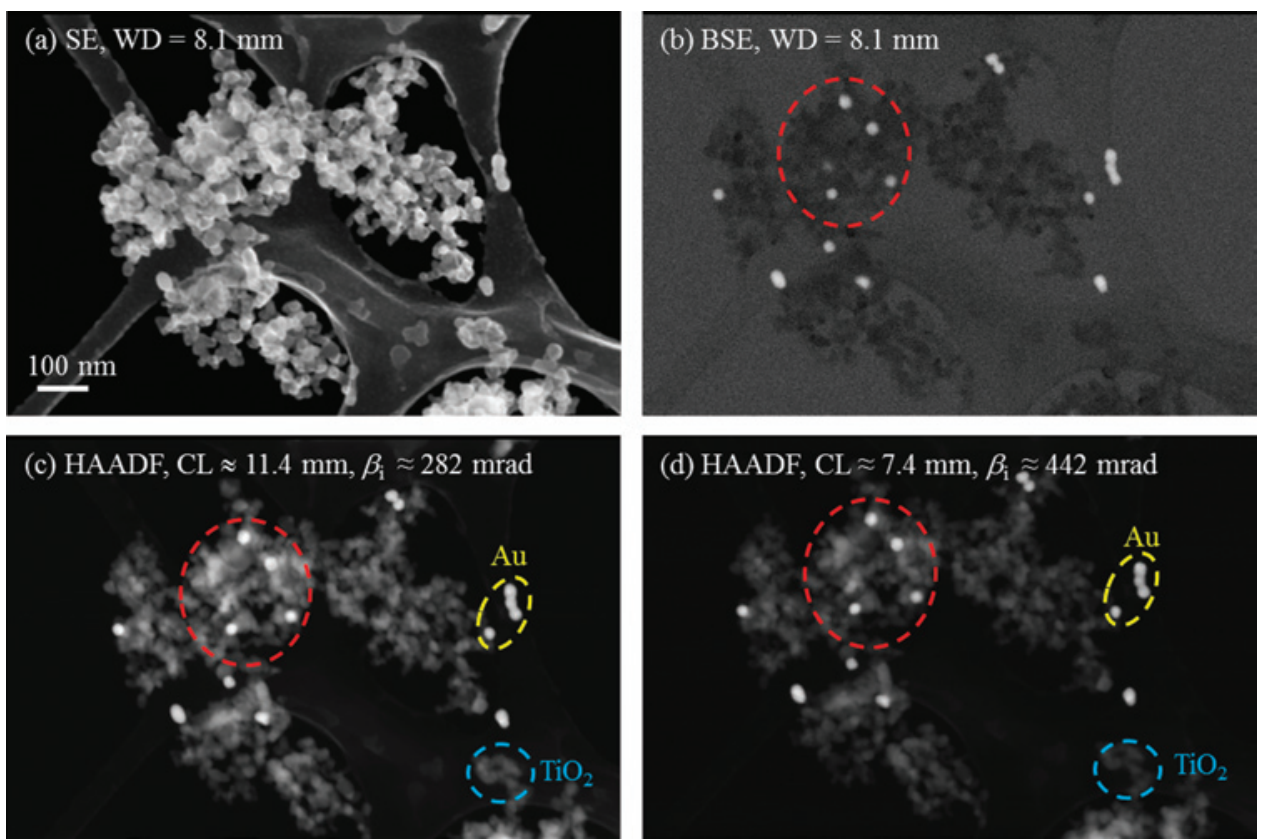

Figure 6: $\mathrm{Au}$ and $\mathrm{TiO}_{2}$ particles on lacey carbon imaged with different detectors: (a) SE image (WD $\left.=8.1 \mathrm{~mm}\right)$, (b) BSE image (WD $=8.1 \mathrm{~mm})$, (c) HAADF STEM image $\left(R_{A i}=3 \mathrm{~mm}, \mathrm{CL}=11.4 \mathrm{~mm}, \mathrm{WD}=8.1 \mathrm{~mm}\right)$, and (d) HAADF STEM image $\left(R_{A i}=3 \mathrm{~mm}, C L=7.4 \mathrm{~mm}, W D=12.1 \mathrm{~mm}\right)$. STEM detector gain settings were unchanged for Figures $6 \mathrm{c}$ and $6 \mathrm{~d}$.

transmitted electron imaging may be worth considering because of the higher probability of forward-scattering than back-scattering. For example, Figure 6 shows images of $\mathrm{Au}$ and $\mathrm{TiO}_{2}$ particles on a lacey carbon substrate recorded using the SE detector (6a), the BSE detector (6b), and the STEM detector in HAADF mode (6c and $6 \mathrm{~d}$ ). The STEM images were collected using the same gain settings at $\mathrm{CL} \approx 11.4 \mathrm{~mm}$ (Figure $6 \mathrm{c}$ ) and $\mathrm{CL} \approx 7.4 \mathrm{~mm}$ (Figure $6 \mathrm{~d}$ ) with an aperture similar to the one shown on the top left of Figure 4a. The STEM and BSE images both show Z-contrast information complementary to the SE image in that isolated Au particles can be discerned from isolated $\mathrm{TiO}_{2}$ particles. Although the BSE image shows the Au particles as bright spots, the STEM images simultaneously show the Au particles (four of which are circled in yellow) and the $\mathrm{TiO}_{2}$ particles, which are generally less bright.
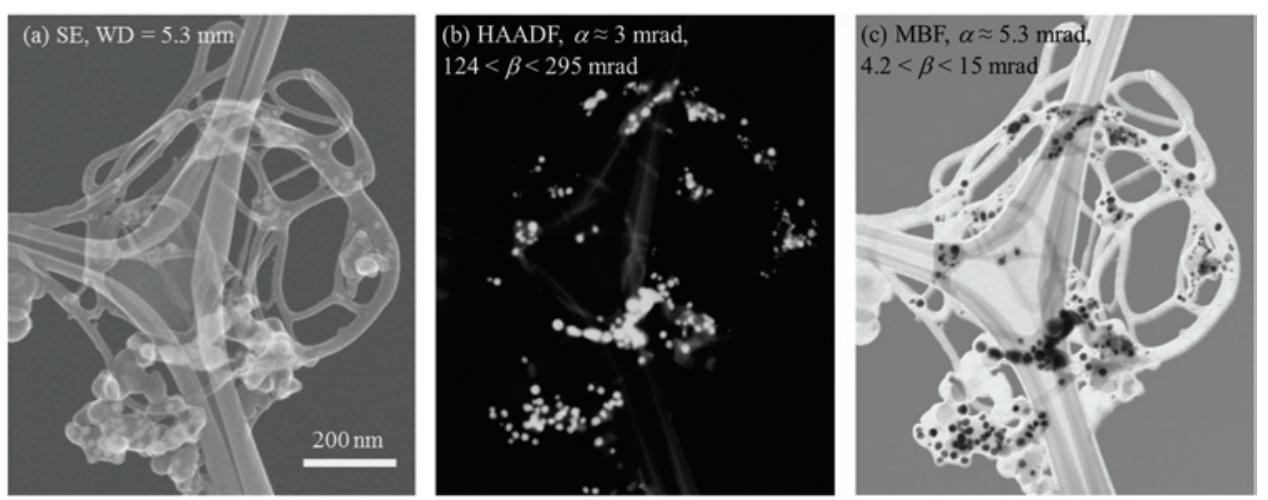

Figure 7: SWCNT bundles with metal catalyst particles and amorphous carbon imaged with (a) the SE detector, (b) in HAADF STEM mode (aperture $R_{\mathrm{Ai}} \approx 0.5 \mathrm{~mm}$ and $R_{\mathrm{Ao}} \approx 1.25 \mathrm{~mm}$ ), and (c) in marginal BF STEM mode (aperture $R_{\mathrm{Ai}} \approx 60 \mu \mathrm{m}$ and $R_{\mathrm{Ao}} \approx 0.22 \mathrm{~mm}$ ). Note that $\beta_{\mathrm{i}}$ is slightly less than the beam convergence semi-angle, $\alpha$, and therefore the image is designated marginal BF.
Depending on the acceptance angles and the sample, STEM image contrast interpretation may or may not be straightforward because a sufficiently large agglomerate of $\mathrm{TiO}_{2}$ particles can elicit the same mass-thickness contrast as a single $\mathrm{Au}$ particle. This effect can be observed in the HAADF (Z-contrast) image of Figure 6c. Within the red dashed circle several regions of strong contrast are visible (both $\mathrm{Au}$ and $\mathrm{TiO}_{2}$ ). The contrast is not particularly amenable to direct visual interpretation. However, Figure $6 \mathrm{~d}$ shows that by moving the sample closer to the STEM detector (that is, reducing the $\mathrm{CL}$ ) the acceptance angle increases, and the contrast between the $\mathrm{Au}$ particles and the $\mathrm{TiO}_{2}$ particles increases. The persistent bright spots can be assigned to the $\mathrm{Au}$ particles. This assignment is supported by the BSE image, which shows bright spots in the same locations as those in Figure 4d.

Catalyst particles in bundled carbon nanotubes. Combining small apertures and long CLs to mix BF and DF signals can elicit useful contrast. For example, Figure 7 shows different ways to discriminate metal catalyst particles from carbon in a highly bundled SWCNT sample. Although some catalyst is visible in the SE image (Figure 7a), the HAADF STEM image (Figure $7 \mathrm{~b}$ ) directly reveals the catalyst particles as the bright spots. The amorphous carbon and SWCNT bundles, however, are generally not visible. In the marginal $\mathrm{BF}$ image (Figure $7 \mathrm{c}$ ), residual catalyst and amorphous carbon, SWCNT bundles, and the carbon substrate can all be observed simultaneously, and the image features are generally sharper than those in Figures $7 \mathrm{a}$ and $7 \mathrm{~b}$. The catalyst particles appear dark in the marginal BF image because the STEM detector aperture only admits electrons scattered into acceptance angles between $\sim 4$ and $15 \mathrm{mrad}$. As observed previously [10], regions of the sample with greater mass-thickness can appear darker than regions with lesser mass-thickness. In this instance, the metallic catalyst particles scatter a significant fraction of electrons through angles larger than $15 \mathrm{mrad}$. Therefore, the signal due to the catalyst will be weak, and the particles will appear dark compared to the rest of the image. Scattering angles associated with the carbon are generally more shallow, and therefore a large fraction of the signal is collected resulting 


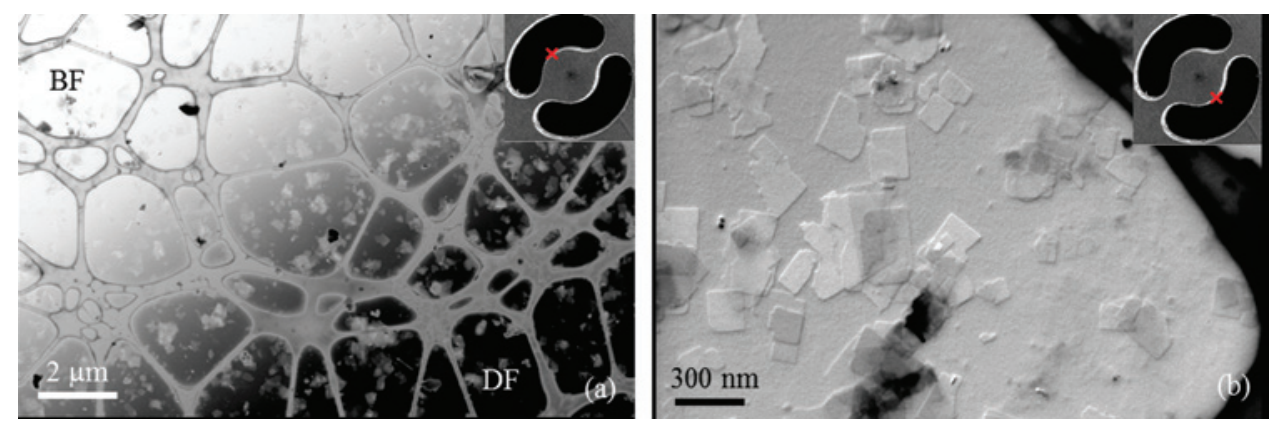

Figure 8: STEM images of 2D exfoliated zeolites with the optic axis positioned at the edge of a small ADF aperture $\left(R_{\mathrm{Ai}}=0.25 \mathrm{~mm}, R_{\mathrm{Ao}}=0.5 \mathrm{~mm}\right)$ : (a) an image simultaneously showing $\mathrm{BF}$ and $\mathrm{DF}$ regions, and the transition region between the two, and (b) an image of the sample in the transition region. The red markers in the insets indicate the optic axis position on the aperture.

in brighter regions in the image. The image background exhibits a moderate level of contrast because electrons in the outer fringe of the incident illumination cone (that is, primary electrons with incident angles between 4.2 and $5.3 \mathrm{mrad}$ ) are able to pass through the aperture and be collected by the STEM detector. Despite the unconventional contrast, discerning the different phases in Figure $7 \mathrm{c}$ is still straightforward.

Exfoliated 2D zeolite sheets. Another way BF and DF signals can be mixed is by moving the STEM detector laterally with respect to the optic axis. For example, Figure 8 shows STEM images of $\sim 3 \mathrm{~nm}$ thick exfoliated zeolite sheets [9] on an ultra-thin carbon/lacey carbon substrate. Here, the detector was moved so the optic axis intersected the edge of a small ADF aperture $\left(R_{\mathrm{Ai}}=0.25 \mathrm{~mm}, R_{\mathrm{Ao}}=0.5 \mathrm{~mm}\right)$ at the point indicated by the red ' $x$ ' in the insets. Figure 8 a shows both $\mathrm{BF}$ and $\mathrm{DF}$ regions, as well as the transition between them. Figure $8 \mathrm{~b}$ shows a higher magnification image of the transition region. The image is different from conventional STEM images in that it appears to show topographic information.

\section{Discussion}

One feature that should be considered when implementing the proposed experimental setup is that by using the SEM sample positioning stage to change the CL, the working distance (WD) must change to maintain focus at the sample. As the WD changes to maintain focus at the sample, the beam convergence angle must also change. For the SEM used here, the beam convergence half-angle can be reasonably estimated as $\alpha \approx 2.53 D_{\mathrm{a}} /(W D+9)$, where $D_{\mathrm{a}}$ is the beam condenser aperture diameter, and $W D$ is the working distance (both with $\mathrm{mm}$ units). The $30 \mu \mathrm{m}$ condenser aperture used here, combined with the $\sim 1-20 \mathrm{~mm} \mathrm{CL}$, enables $\sim 2.6<\alpha<\sim 7.5 \mathrm{mrad}$. This can be advantageous in a microscope that does not directly enable beam convergence angle control: more parallel illumination can be obtained by employing a long WD and/or a smaller beam condenser aperture, and more convergent illumination can be obtained by using a short WD and/or a larger beam condenser aperture. Knowledge of this feature is useful for controlling which scattering mechanisms contribute to image contrast. For example, small-angle coherent scattering (due on the combination of sample, STEM detector, primary electron beam condenser apertures, WD, CL, and primary electron energy. Each of these parameters should be carefully considered when collecting and interpreting images. If the effects of changing beam convergence angle are not desired for a particular experiment, then the sample can be held stationary and the STEM detector $x y z$-positioning stage can be used to change the CL by $\sim 10 \mathrm{~mm}$.

Most commercially available STEM detectors have inherently large acceptance angle ranges, and therefore multiple contrast mechanisms will likely contribute to image contrast, particularly at small acceptance angles. For example, solid-state STEM detectors generally employ a BF detector with an acceptance angle significantly larger than the beam convergence angle. The STEM detector used here has an integrated $100 \mu \mathrm{m}$ aperture for $\mathrm{BF}$ imaging that can enable acceptance half-angles up to $50 \mathrm{mrad}$. This is significantly larger than the $7.5 \mathrm{mrad}$ beam convergence angle available with the $30 \mu \mathrm{m}$ condenser aperture. In conventional TEM parlance, this means multi-beam imaging conditions likely contribute to image contrast. While combined contrast mechanisms can lead to useful images as demonstrated by Figure $7 \mathrm{c}$, it can also complicate image interpretation. A significant advantage to the modular aperture approach described here is that very narrow acceptance angle ranges can be obtained over a wide range of scattering angles, and, therefore, electrons scattered by different mechanisms can be selected in controlled manner. In the long-term, this ability should improve the understanding of transmission imaging in an SEM, make image interpretation straightforward, and lead to rigorous quantitative analyses with reduced uncertainty. In the short term, it is unclear why Figures $5 \mathrm{a}$ and $5 \mathrm{~d}$ exhibit poor resolution and weak contrast compared to the other images. These phenomena have been consistently observed with diverse samples at very short camera lengths with and without the aperture system in place. It is unlikely that the reduced imaging performance is due to the aperture system.

In closing, an operational guideline is recommended for new STEM-in-SEM users. Most modern SEMs enable large beam shift capabilities that compensate for moderately coarse sample stage positioning steps. Using the beam 
shift to center a sample feature on the optic axis can lead to complex image contrast that can change in unexpected ways because the optic axis moves with respect to the STEM detector. Depending on the amount of applied beam shift, effects similar to the ones observed in Figure 8 can be obtained, albeit unexpectedly. For example, although the user may have carefully chosen a set of apertures to obtain Z-contrast, using the beam shift skews the acceptance angle, and contrast other than that due to incoherent Rutherford scattering may be present in the image. To avoid this potential complication, the sample stage should be used to center features of interest.

\section{Conclusion}

A modular detection system and new sample holder for transmission-SEM imaging have been described. By combining different masks/apertures and using the sample positioning stage to adjust the camera length, comprehensive detector acceptance angle control is possible. The acceptance angle control enabled here is unavailable in commercial SEM detectors: thin annular detection schemes are feasible, separating diffraction contrast from Z-contrast is feasible, and BF-DF transition region signals can be used to obtain unusual image contrast. Although the system has not been fully explored, several examples show that it is a promising approach to extending the imaging capabilities of almost any SEM.

\section{Disclaimers}

This contribution is by NIST, an agency of the US government, and is not subject to copyright in the United States. Commercial instruments, equipment, or materials are identified only in order to adequately specify certain procedures. In no case does such identification imply recommendation or endorsement by NIST, nor does it imply that the products identified are the best available for the purpose.

\section{References}

[1] T Klein et al., Adv Imag Elect Phys 171 (2012) 297-356.

[2] RJ Woolf et al., J Phys E: Sci Instrum 5 (1972) 230-33.

[3] U Valdre et al., Ultramicroscopy 15 (1984) 109-18.

[4] E Oho et al., J Elec Microsc Tech 5 (1987) 51-58.

[5] M Haider et al., Ultramicroscopy 54 (1994) 41-59.

[6] A Khursheed et al., Rev Sci Instr 74 (2003) 134-40.

[7] E Buhr et al., Meas. Sci Tech 20 (2009) 084025.

[8] B Jacobson et al., Proc of SPIE 9376 (2015) 93760K.

[9] K Varoon et al., Science 334 (2011) 72-75.

[10] J Holm and R Keller, Ultramicroscopy 167 (2016) 43-56.

[11] S Findlay et al., Ultramicroscopy 110 (2010) 902-23.

[12] J Cowley, J Elec Microsc 50 (2001) 147-55.

[13] J Cowley et al., Ultramicroscopy 58 (1996) 18-24.

[14] OL Krivanek et al., Nature 464 (2010) 571-74.

[15] DE Jesson and SJ Pennycook, Proc R Soc Lond A 449 (1995) 273-93.

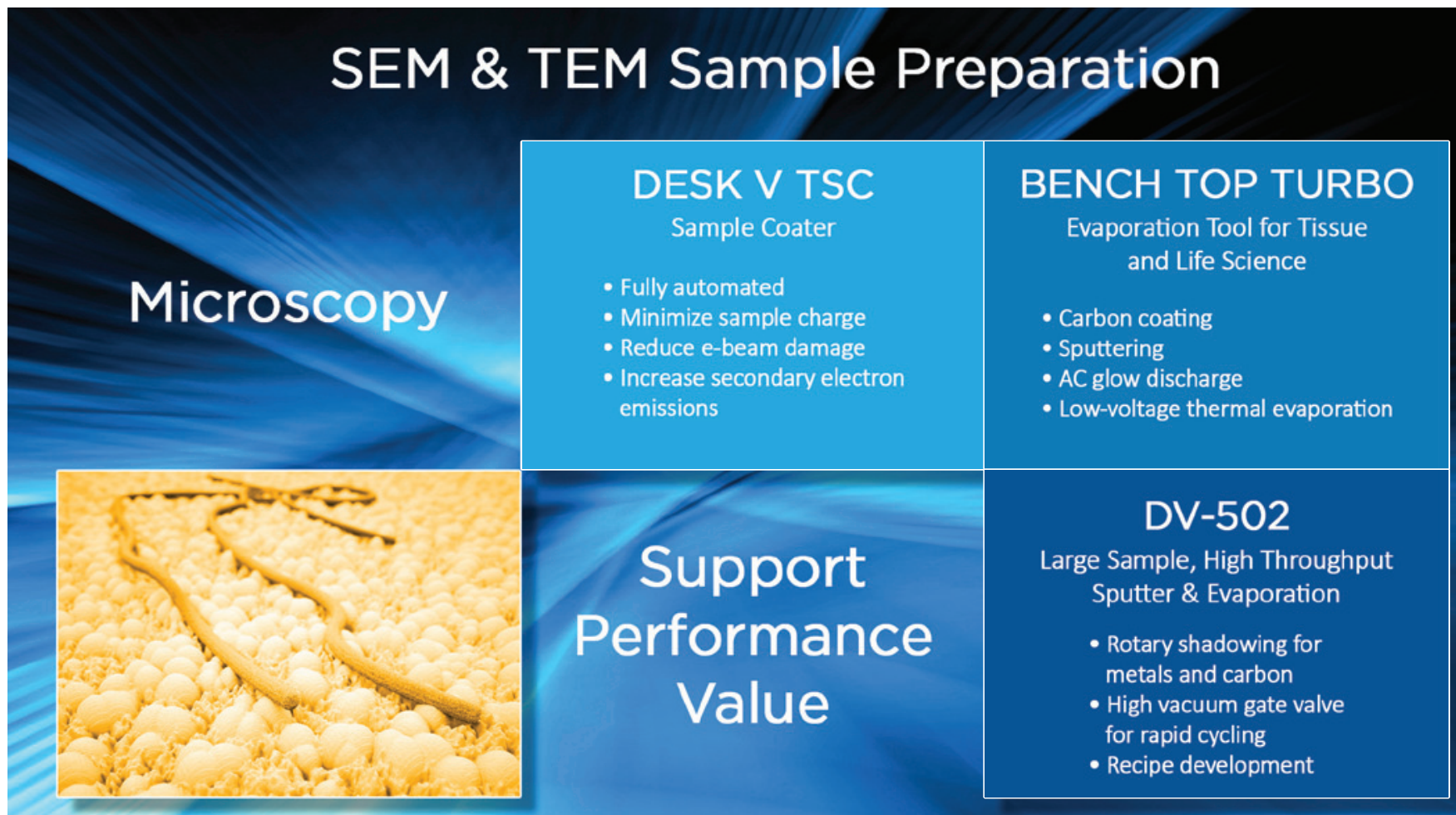




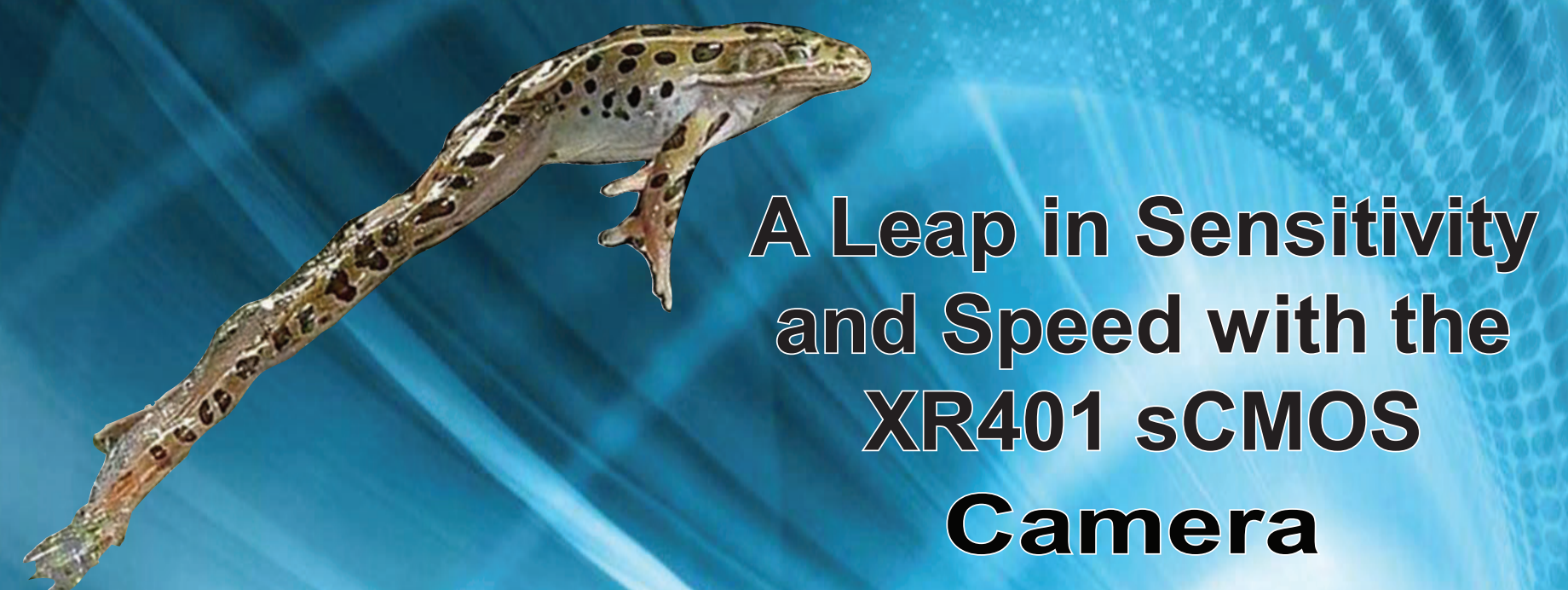

- Cryo TEM

- Low Dose TEM

- Diffraction

- In-Situ TEM

- Extraordinary speed

- Non-Blooming Sensor $0<2$ e/pixel noise

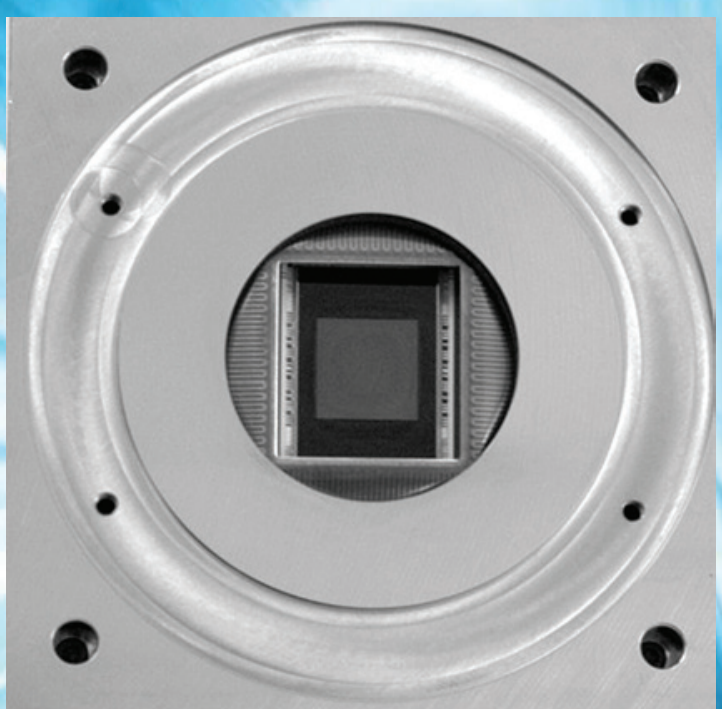

Adenovirus

Dr. Cameron Ackerley

The Hospital for Sick Children

\section{and Speed with the XR401 sCMOS \\ Camera}

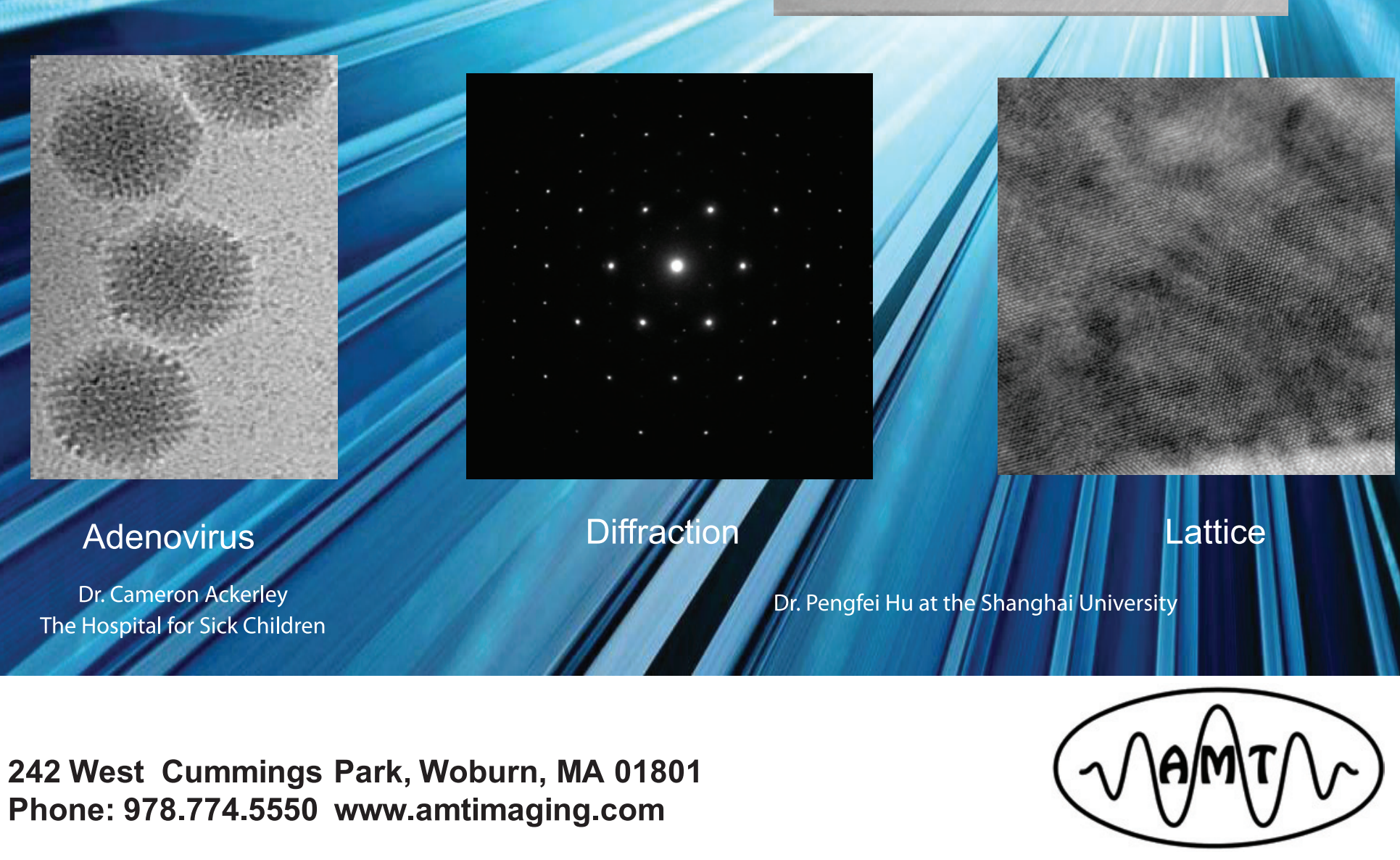

